\title{
7. Histological Study of the Distribution of Cervical Sensory Nerves
}

\author{
Hideo Aoki and Yuko TokUnaga \\ Second Surgical Department, Yamaguchi Medical College
}

Headache is undoubtedly the commonst complaint of patients in the general medicine. Recent advances in the treatment of chronic headache have reported markedly good result, but the patients complained many types of chronic headache such as posttraumatic headache, atypical facial pain, tension headlache, migraine etc. increasing gradually.

The great auricular neurotomy and block with alcohol of this nerve were reported by Mason, W. R. as a new procedures of the surgical treatment for tic douleureux. In our clinic these procedures, except that localized anesthetic agent was used as blocking agent instead of alcohol, have bcen carried about 80 cases complained various type of headache as mentioned above. In about 80 percent of these cases we were successful in releaving the pain. In this series two cases of the glossopharyngeal neuralgia was included. It is interest that these two patients were relieved dramatically from the intractable severe pain by block and section of this nerve respectively.

From this clinical experience, it appears that sensory fibers from the cervival nerve are carried into trigeminal and glossopharyngeal area. Consequently following experiments was carried out using dog. The cervical nerves (C II, III) were dissected in the subcutaneous region, and it was demonstrated by the histological examination that degenerative nerve fibers were observed mandibular or buccal region, particularly in pharyngeal region.

In conclusion overlapping of the sensory innervation was proved between the cervical nerves (C II, III,) and the cranial nerves (V, VII, and IX) in the head, face and pharynx. 